\title{
Dendritic cell-targeted vaccines - hope or hype?
}

\section{Wolfgang Kastenmüller, Kathrin Kastenmüller, Christian Kurts and Robert A. Seder}

Abstract|The development of an effective vaccine that elicits a strong and durable T cell response against intracellular pathogens and cancer is a challenge. One strategy to enhance the effectiveness of vaccination is by targeting dendritic cells (DCs). In this Opinion article, we discuss existing DC-targeting approaches that induce adaptive immunity. We highlight the crucial issues that need to be addressed to move the field forward and discuss whether targeting DCs could be better than current vaccine approaches.

Historically, the earliest successful vaccines used live attenuated pathogens, such as vaccinia virus, to induce protective immunity. Subsequently, inactivated viruses, subunit vaccines and protein-polysaccharide conjugate vaccines - which all induce antibodymediated immunity - have been used to protect against different viral and bacterial infections ${ }^{1}$. However, there is currently no effective vaccine for several diseases - such as HIV, malaria and tuberculosis - in which $\mathrm{T}$ cells can have a protective role in the presence or absence of antibodies. Researchers now try to optimize both $\mathrm{B}$ cell and $\mathrm{T}$ cell responses to broadly attack pathogen ${ }^{2-4}$. Dendritic cells (DCs) - which are the most potent antigen-presenting cells - induce the clonal expansion of $\mathrm{T}$ cells ${ }^{5}$, and are therefore an important cell type to consider targeting in the development of vaccines that induce effective and durably protective $\mathrm{T}$ cell immunity. In addition, such DC-targeting vaccines could be used to treat other diseases, such as cancer, that also require $\mathrm{T}$ cell immunity.

The first attempts to use DCs to induce adaptive immunity in humans involved the adoptive transfer of in vitro-cultured DCs loaded with antigens, which was used as immunotherapy against cancer ${ }^{6}$. Although data from $>10$ years of clinical trials indicate that ex vivo antigen-loaded DC-based vaccines are safe and can induce tumour-specific $\mathrm{CD} 4^{+} \mathrm{T}$ cells and cytotoxic T lymphocytes (CTLs) in humans, durable tumour regression has only been observed in a few patients ${ }^{7}$. Instead, most patients showed minimal adaptive immunity, displayed no clinical signs of tumour control and did not have prolonged survival ${ }^{8}$.
Recently, investigators have used short-term cultured DCs or naturally circulating DCs to improve the function of these cells and the clinical outcome of cancer therapy ${ }^{9,10}$. Nevertheless, the use of ex vivo-cultured DCs is labour-intensive, expensive and needs to be individualized to each patient; this approach will not be discussed further here. In this Opinion article, we take a critical look at various DC-targeting approaches, discuss their scope and give a perspective on the steps that need to be taken to improve the ability of protein-based vaccines to elicit T cell immunity through DC activation.

\section{Adjuvants for DC vaccines}

An efficient way to activate $\mathrm{T}$ cells is through the presentation of antigen by DCs that simultaneously undergo innate immune activation ${ }^{11,12}$. Accordingly, T cells primed by a complex pathogen often elicit the most robust responses; for example, viruses can efficiently prime CTL responses by promoting synchronous activation of and antigen presentation by $\mathrm{DCs}^{13}$. Thus, one option to mimic the efficiency of viralbased vaccines is by linking proteins to pattern recognition receptor (PRR) ligands to create antigen-PRR-ligand conjugate vaccines, which ensures that antigen processing and stimulation occur simultaneously in the same DC.

Antigen-PRR-ligand conjugate vaccines. When designing an antigen-PRR-ligand conjugate vaccine, the choice of antigen is determined by the pathogen, whereas the selection of PRR ligands that could be used as adjuvants is extensive $\mathrm{e}^{14,15}$ and depends on the desired immune response (for example, a T helper $1\left(\mathrm{~T}_{\mathrm{H}} 1\right)$ cell, $\mathrm{T}_{\mathrm{H}} 2$ cell or $\mathrm{T}_{\mathrm{H}} 17$ cell response) and the PRR expression profile of the targeted DCs (TABLE 1). Thus, a specific set or a wide range of DCs can be engaged depending on the Toll-like receptor (TLR) ligand that is used as an adjuvant.

The increased efficiency of antigen-TLRligand conjugate vaccines to generate an adaptive immune response compared with free antigen and ligand was first demonstrated in mouse immunization studies using CpG (which is a TLR9 ligand) and the ovalbumin (OVA) antigen ${ }^{16-18}$. The conjugate vaccine elicited more potent $\mathrm{T}_{\mathrm{H}} 1$ cell and IgG2a antibody responses, and cross-primed CTLs at lower doses compared with the administration of OVA and free CpG. However, the efficacy of TLR9 ligands might be limited in humans, as the expression of TLR9 is more restricted in the human DC subsets that are crucial for antigen presentation than in the corresponding cells in mice (TABLE 1). By contrast, TLR7 and TLR8 are expressed across all human DC subsets; however, the rapid dissolution of TLR7-TLR8 agonists from the injection site limits their ability to prime T cells in the draining lymph nodes, thereby reducing their ability to induce protective adaptive immunity ${ }^{19}$. Nevertheless, studies in mice and non-human primates (NHPs) showed that this rapid dissolution could be prevented if the HIV Gag protein was conjugated to a TLR7-TLR8 agonist, which enabled prolonged antigen presentation and innate immune stimulation in the draining lymph nodes, and elicited potent $\mathrm{T}$ cell responses ${ }^{20,21}$.

The efficacy of several antigen-TLRligand conjugate vaccines has been tested in mice and all of these elicited a stronger immune response than simple mixtures of antigen and adjuvant, but few of these vaccines have advanced to studies in NHPs or humans to evaluate their efficacy (TABLE 2). Overall, the conjugation platform enables multiple DC subsets with different specialized functions to capture antigen and become activated.

The immune system has evolved so that DC subsets with different specialized functions collaborate to induce a complex pathogen-specific immune response and therefore, engaging multiple DC subsets may be superior to targeting only one DC subset. This is important to consider for vaccine development; is the engagement of multiple DCs - by using a well-formulated protein and adjuvant vaccine - or the targeting of multiple DC receptors required to optimize broad-based T cell immunity? 
Table 1 | Expression patterns of receptors in mouse and human DC subsets

\begin{tabular}{|c|c|c|c|c|c|c|}
\hline \multirow[t]{2}{*}{ Receptor } & \multicolumn{3}{|c|}{ Mouse DC subsets } & \multicolumn{3}{|c|}{ Human DC subsets } \\
\hline & $\mathrm{CD}_{11 b^{+}}$ & $\mathrm{CD} 8 \mathrm{a}^{+}$or $\mathrm{XCR} 1^{+}$ & $\mathrm{pDC}$ & BDCA1 $^{+}$ & $\mathrm{BDCA}{ }^{+}$or $\mathrm{XCR} 1^{+}$ & pDC \\
\hline \multicolumn{7}{|c|}{ Innate receptor } \\
\hline TLR3 & - & + & - & - & + & - \\
\hline TLR4 & $-/+^{*}$ & - & - & + & - & - \\
\hline TLR7 & - & - & + & + & - & + \\
\hline TLR9 & + & + & + & - & - & + \\
\hline NLRP3 & + & - & - & + & $?$ & $?$ \\
\hline NOD1 & + & - & - & + & $?$ & $?$ \\
\hline \multicolumn{7}{|c|}{ Targeting receptor } \\
\hline XCR1 & - & + & - & - & + & - \\
\hline CLEC9A & - & + & + & - & + & - \\
\hline CLEC12A & - & + & + & + & + & + \\
\hline CD205 & - & + & - & + & + & - \\
\hline CD207 & - & $-1+^{*}$ & - & - & - & - \\
\hline $\mathrm{MHCII}^{\ddagger}$ & + & + & + & + & + & + \\
\hline $\mathrm{CD} 40^{\ddagger}$ & + & + & + & + & + & + \\
\hline CD11c & + & + & - & + & + & - \\
\hline Fc $\gamma R$ & + & + & + & + & + & + \\
\hline
\end{tabular}

+, expressed; -, low or no expression; ?, expression level is unknown; BDCA, blood DC antigen; CLEC, C-type lectin domain family member; DC, dendritic cell; Fc $\gamma \mathrm{R}$, Fc receptor for IgG; $\mathrm{MHCII}, \mathrm{MHC}$ class II; NLRP3, NOD-, LRR- and pyrin domain-containing 3; NOD1, nucleotide-binding oligomerization domain-containing protein 1; pDC, plasmacytoid DC; TLR, Toll-like receptor; XCR1, XC-chemokine receptor $1 .{ }^{*}$ Mouse strain dependent. ${ }^{\ddagger}$ Activation dependent.

\section{Targeting antigens to DCs}

The most widely studied approach to activate $\mathrm{T}$ cells involves the selective targeting of DC-specific endocytic receptors by linking the relevant antigens to antibodies or ligands. Following internalization by the targeted DCs, the intracellular routing of these complexes depends on the specific receptor, and has important functional consequences with regard to antigen presentation and T cell stimulation (FIG. 1). As monoclonal antibodies are now used in a large number of patients with a variety of diseases, they offer the advantage of a safe, scalable and standardized vaccine that targets DCs within their natural environment in vivo. In addition, targeting antigens to DC-specific receptors may reduce the required vaccine dose. Even more importantly, this reduces the proportion of the vaccine dose that ends up in non-target cells, and thus reduces potential adverse effects. However, whether avoiding antigen capture by non-target cells has any functional relevance or negatively affects the success of vaccination is still not clear.

DC targeting can also be used to affect the quality of the ensuing immune response. To date, $>100$ DC-targeting studies have been published (these are summarized in
TABLE 2 and Supplementary information S1 (table)). The first DC-targeting studies in mice were aimed at $F_{c} \gamma$ receptors ( $F c \gamma R s$ ), MHC class II molecules and CD40 (REFS 22-24), and showed that antigen targeting improves both humoral and cellular adaptive immunity, with the former not requiring the administration of additional adjuvants. During the last decade, research has focused on C-type lectins and, in particular, CD205 (also known as LY75) has been extensively studied in mice, NHPs and humans. Studies on CD205 have revealed several important facts: targeting antigens to CD205 in the absence of adjuvants was shown to cause tolerance; the TLR3 ligand poly I:C was the most effective adjuvant for inducing potent $\mathrm{CD}^{+} \mathrm{T}$ cell and low-level CTL responses; and potent CTL responses were observed using OVA combined with adjuvants but, importantly, the magnitude of the $\mathrm{CD}^{+} \mathrm{T}$ cell response was far lower with other antigens, such as HIV Gag. Moreover, CD205 targeting induced only low-level CTL responses in NHPs in studies using the malaria circumsporozoite protein or HIV Gag ${ }^{25}$. Importantly, these low CTL responses were markedly increased after a heterologous boost with a viral vaccine encoding HIV Gag. These data highlight the importance of combining different vaccine approaches for optimizing CTL responses, as discussed below. With regard to $\mathrm{CD} 4^{+}$ T cells, a non-DC-targeted HIV Gag protein-based vaccine induced a similar $\mathrm{CD}^{+} \mathrm{T}$ cell response to the CD205-targeted vaccine in NHPs, but led to the production of higher quality antibodies ( 10 -fold increased avidity). Although mouse studies suggest that CD205 targeting could be effective at eliciting $\mathrm{CD} 4^{+} \mathrm{T}$ cell responses, studies in NHPs showed no difference in $\mathrm{T}_{\mathrm{H}} 1$ cell priming and antibody responses when a more physiological antigen, such as HIV Gag, was used (see Supplementary information S1 (table)).

As mouse DCs that express CD8a are superior at cross-presentation, targeting this DC subset could theoretically be advantageous for inducing $\mathrm{CD} 8^{+} \mathrm{T}$ cell responses. The human orthologue of this DC subset lacks CD8 $\alpha$, but instead expresses C-type lectin domain family 9 member A (CLEC9A; also known as DNGR1), XC-chemokine receptor 1 (XCR1) and blood DC antigen 3 (BDCA3; also known as CD141 and thrombomodulin) ${ }^{26-29}$. CLEC9A and XCR1 seem to be similarly specific for targeting the CD $8 \alpha^{+}$DC subset in mice, and could be used to eradicate established melanomas (R. A. Kroczek, personal communication) ${ }^{30}$. Additionally, CLEC9A targeting can modulate the in vivo differentiation of $\mathrm{CD} 4^{+} \mathrm{T}$ cells into $T_{H} 1$ cells, $T_{H} 17$ cells or regulatory $T$ cells using poly I:C, curdlan or adjuvantfree immunization, respectively ${ }^{31}$. Overall, the ability of CLEC9A and XCR1 to target $\mathrm{CD} 8 \alpha^{+}$DCs in mice makes these receptors perhaps the most promising candidates for the induction of CTL responses. Hence, it is crucial to test whether targeting to these receptors can efficiently induce cross-priming in NHPs and ultimately in humans.

Integrins that are highly expressed by macrophages and DCs, such as CD11b (also known as integrin- $\alpha \mathrm{M}$ and ITGAM) and CD11c (also known as integrin- $\alpha \mathrm{X}$ and ITGAX), have also been used for DC targeting (see Supplementary information S1 (table)). A detoxified version of the adenylyl cyclase CyaA from Bordetella pertussis, which binds to CD11b, has been extensively studied and was shown to induce protective immune responses against viral infections and cancer (see Supplementary information S1 (table)). Importantly, a CyaA construct expressing the full-length HIV Tat protein induced HIV Tat-specific CD4 ${ }^{+} \mathrm{T}$ cell and antibody responses in mice and in $\mathrm{NHPs}^{32,33}$. Further investigation is required to determine whether robust $\mathrm{T}$ cell responses can 


\section{Table 2 | The efficacy of antigen-PRR-ligand conjugate vaccines}

\begin{tabular}{|c|c|c|c|c|c|c|}
\hline $\begin{array}{l}\text { Targeted } \\
\text { receptor }\end{array}$ & Organism & Delivered antigen & Adjuvant & Immune responses stimulated & Efficacy test & Refs \\
\hline TLR9 & Mouse & OVA & $\mathrm{CpG}$ & CD8 ${ }^{+} \mathrm{T}$ cells (in vivo) & Tumour & 16 \\
\hline TLR9 & Mouse & OVA & $\mathrm{CpG}$ & CD8 ${ }^{+} \mathrm{T}$ cells (in vitro) & NA & 59 \\
\hline TLR9 & Mouse & Amb a 1 & $\mathrm{CpG}$ & Antibodies and $\mathrm{T}_{H} 1$-type response (in vivo) & Allergy & 18 \\
\hline TLR9 & Mouse & gp120 & $\mathrm{CpG}$ & Antibodies, and $\mathrm{CD} 4^{+}$and $\mathrm{CD} 8^{+} \mathrm{T}$ cells (in vivo) & NA & 60 \\
\hline TLR9 & Mouse & OVA & $\mathrm{CpG}$ & $\mathrm{CD} 8^{+} \mathrm{T}$ cells (in vivo) & Pathogen & 61 \\
\hline TLR9 & Mouse & $\begin{array}{l}\beta \text {-galactosidase and } \\
\text { gp120 }\end{array}$ & $\mathrm{CpG}$ & Antibodies and $C D 8^{+} T$ cells (in vivo) & NA & 17 \\
\hline TLR7-TLR8 & Mouse & HIV Gag & $3 \mathrm{M}-012$ and $\mathrm{CpG}$ & $\mathrm{CD} 4^{+}$and $\mathrm{CD} 8^{+} \mathrm{T}$ cells (in vivo) & NA & 19 \\
\hline TLR7-TLR8 & NHP & HIV Gag & $3 \mathrm{M}-012$ and $\mathrm{CpG}$ & Antibodies, and $\mathrm{CD} 4^{+}$and $\mathrm{CD} 8^{+} \mathrm{T}$ cells (in vivo) & NA & 21 \\
\hline TLR7-TLR8 & Mouse & OVA & $3 \mathrm{M}-012$ & $\mathrm{CD} 4^{+}$and $\mathrm{CD} 8^{+} \mathrm{T}$ cells (in vivo) & Pathogen & 20 \\
\hline
\end{tabular}

ESAT-6, early secreted antigenic target of $6 \mathrm{kDa}$ (also known as EsxA); LLO, listeriolysin O; NA, not applicable; NHP, non-human primate; OVA, ovalbumin; p60, murein hydrolase; Pam2Cys, S-[2,3-bis(palmitoyloxy)propyl]cysteine; PRR, pattern recognition receptor; Rv1411c, a Mycobacterium tuberculosis lipoprotein functioning as a TLR2 agonist; $T_{H} 1$, T helper 1 ; TLR, Toll-like receptor.

be induced by this approach and others using targets such as mannose receptor 1 (also known as CD206) or DC-specific ICAM3-grabbing non-integrin (DC-SIGN; also known as CD209; see Supplementary information S1 (table)).

Comparative studies of in vivo DC targeting Several recent studies have directly compared the efficiency of different DC-targeting approaches (TABLE 3). These studies are crucial, as they provide the necessary benchmarking and rationale for translating these approaches into human trials ${ }^{34}$. Studies in mice that compared antigentargeting to the DC receptors CD205, CD207 (also known as CLEC4K), DC inhibitory receptor (DCIR; also known as CLEC4A), CLEC9A, TREML4 and DCIR2 (also known as CLEC4A4) revealed that CD205, CD207 and CLEC9A were the most potent targets for inducing CTL responses against HIV Gag ${ }^{35-37}$. These findings were independently confirmed and also compared with CLEC12A, which proved less efficient ${ }^{38}$. Together, these studies showed that in addition to targeting antigen to DCs, prolonged antigen presentation correlated with the strength of CTL responses.

In another study, CD205 targeting with OVA was not superior to targeting MHC class II, CD11c, CD40, TLR2, or Fc $\gamma$ RII and $\mathrm{F}_{\mathrm{c}} \gamma \mathrm{RIII}^{39}$. Moreover, when in vivo $\mathrm{T}$ cell proliferation was used as a readout for $\mathrm{T}$ cell priming, CD11c targeting was 3-4-fold more efficient than CD205 targeting ${ }^{39}$. In a follow-up mouse study, in vivo antibody responses were compared for 13 different targeting antibodies - including those specific for MHC class II, CD11a (also known as ITGAL), CD11b, CD11c, CD205, DCIR2 and CD40 - and showed that CD11c targeting was the most potent ${ }^{40}$. The authors speculated that CD11c targeting triggers an unknown adjuvant effect in vivo, because the administration of CD11c-specific monoclonal antibodies without antigen enhanced the humoral immune response that was induced by other targeting monoclonal antibodies. In summary, CD11c targeting seems to be very efficient at inducing CTL and antibody responses in mice. However, the adjuvant effect needs to be discriminated from the ability to deliver antigen to the DC, and whether targeting CD11c is as efficient when more physiologically relevant antigens are used requires further investigation.

Many in vitro studies with human DCs suggest that DC targeting can influence both the extent and quality of the $\mathrm{T}$ cell response. The efficiency of CTL crosspriming depends on the intracellular routing of the receptor-antigen-antibody complexes. A comparison of $\mathrm{BDCA}^{+}$ DC-targeting in vitro - using peptides such as those derived from influenza virus, cytomegalovirus and the cancer-testis antigen NY-ESO-1 (also known as CTAG1) - via CD205, mannose receptor $1, \mathrm{CD} 11 \mathrm{c}$ and CD40 showed that targeting CD40 was most efficient at activating CTLs (TABLE 3). Routing of the internalized complex to early endosomes led to slow antigen release, which enabled prolonged antigen presentation and T cell stimulation ${ }^{41-43}$. Other studies analysed $\mathrm{CD}^{+}{ }^{+} \mathrm{T}$ cell responses after DC-targeted delivery of the antigens haemagglutinin 1 or prostate-specific antigen ${ }^{44}$ to lectin-like oxidized LDL receptor 1 (LOX1; also known as OLR1), dectin 1 (also known as CLEC7A), DC-SIGN and DC-asialoglycoprotein receptor (DC-ASGPR; also known as CLEC10A, MGL and CD301). These studies showed that antigen targeting to DC-ASPGR induced interleukin-10 (IL-10)-producing $\mathrm{CD}^{+} \mathrm{T}$ cells, whereas antigen targeting to LOX1 induced an interferon- $\gamma$ (IFN $\gamma$ ) response $^{44}$. Importantly, these results were confirmed in NHPs, which showed that antigen targeting either LOX1 or DC-ASGPR led to the induction of an IFN $\gamma$ and IL-10 response, respectively, and the proliferation of antigen-specific $\mathrm{CD}^{+}$ $\mathrm{T}$ cells. DC-ASGPR is a $\mathrm{Ca}^{2+}$-dependent type II transmembrane lectin that is localized in early endosomes ${ }^{45}$. DC-ASGPR targeting led to the activation of extracellular signal-regulated kinase (ERK) in and production of IL-10 by the targeted DCs; this, in turn stimulated the differentiation of $\mathrm{CD}^{+} \mathrm{T}$ cells into IL-10-producing suppressor cells ${ }^{44}$. These studies provide important examples of how the inflammatory milieu created by the targeting receptor itself alters the ensuing immune response. 


\section{Intracellular routing}

Receptor that routes cargo to early endosomes upon binding to the targeted vaccine
Receptor that routes cargo to late endolysosomes upon binding to the targeted vaccine

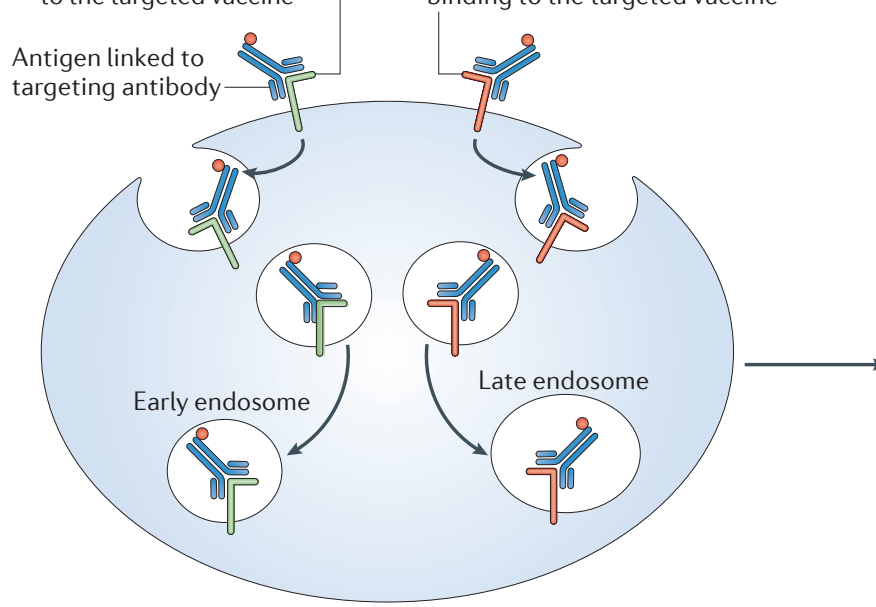

\begin{tabular}{|c|c|c|}
\hline Receptor & $\begin{array}{l}\text { Early endosomal } \\
\text { compartment }\end{array}$ & $\begin{array}{l}\text { Late endosomal } \\
\text { compartment }\end{array}$ \\
\hline CD205 & No & Yes \\
\hline CD207 & Yes & No \\
\hline Mannose receptor 1 & Yes & No \\
\hline DC-SIGN & Yes (ligand dependent) & Yes (ligand dependent) \\
\hline CLEC9A & Yes & No \\
\hline DCIR2 & No & Yes \\
\hline CLEC12A & Not investigated & Not investigated \\
\hline DC-ASGPR & No & Yes \\
\hline Dectin 1 & No & Yes \\
\hline CD11c & Not investigated & Not investigated \\
\hline CD11b & Not investigated & Not investigated \\
\hline MHC class II & No & Yes \\
\hline CD40 & Yes & No \\
\hline$F_{C \gamma} R$ & No & Yes \\
\hline XCR1 or XCL1 & Not investigated & Not investigated \\
\hline
\end{tabular}

$\begin{array}{lccc}\text { Stimulates CD4 } & \text { T cells } & \text { Stimulates CD8 } & \text { T cells } \\ \text { In vitro } & \text { In vivo } & \text { In vitro } & \text { In vivo } \\ + & + & + & ++ \\ + & & & + \text { +- (human) } \\ + & + & + & ++ \\ + & + & + & + \\ + & + & + & + \\ + & + & + & ++ \\ + & ++ & + & + \\ + & ++ & + & + \text { +- } \\ + & ++ & + & + \text { - } \\ + & ++ & + & + \\ + & + & + & ++ \\ + & ++ & + & + \\ + & + & + & + \\ + & + & + & +\end{array}$

Figure 1 Antigen presentation and intracellular routing. Targeting antigens via surface receptors leads to internalization of the receptor together with its cargo. The intracellular routing upon internalization depends on the receptor and has important functional consequences with regard to antigen presentation. Most receptors are routed to late endolysosomes in which antigen is quickly degraded and efficiently presented on $\mathrm{MHC}$ class II molecules to CD4 ${ }^{+} \mathrm{T}$ cells. Some receptors route their cargo to early endosomes in which antigen is slowly digested, leading to prolonged $\mathrm{MHC}$ class I presentation to $\mathrm{CD}^{+} \mathrm{T}$ cells $^{42,43,52}$. Thicker arrows indicate that a

pathway of antigen presentation is more efficient. The table summarizes the preferential intracellular routing of receptors to early and late endosomal compartments, and the stimulation of T cells in vitro and/or in vivo. Unless otherwise indicated, the table refers to data from mice. + , intermediate stimulation of T cells; ++, strong stimulation of T cells; +/-, low stimulation of T cells; CLEC, C-type lectin domain family member; DC-ASGPR, DC-asialoglycoprotein receptor; DCIR2, dendritic cell inhibitory receptor 2; DC-SIGN, DC-specific ICAM3-grabbing non-integrin; Fc $\gamma$ R, Fc receptor for IgG; XCL1, XC-chemokine ligand 1; XCR1, XC-chemokine receptor 1.

\section{A scope for DC targeting}

DC targeting to induce humoral immunity. Most currently approved vaccines consist of a pathogen protein combined with an adjuvant, and these vaccines primarily mediate protection by raising antibodies against the infectious agent. Thus, an important question is whether DC-targeted approaches can induce 'better' antibody responses than current protein-based vaccines ${ }^{46}$ (TABLES 2,3) (also see Supplementary information S1 (table)). Studies in mice have shown that some DC-targeting approaches induce an antibody response even in the absence of an adjuvant (TABLE 3) (also see Supplementary information S1 (table)). However, one potential caveat is that despite antibody induction, the absence of an adjuvant might cause $\mathrm{T}$ cell tolerance. Furthermore, linking an antigen to an antibody by conjugation or expressing the antigen in a targeted antibody may alter its conformation, which may impair the induction of neutralizing antibodies by vaccines for which the structure of the immunogen is crucial, such as HIV and universal influenza vaccines. Thus, unless targeted vaccines can alter the quality or duration 


\section{Table 3 | Comparative studies of in vivo DC targeting}

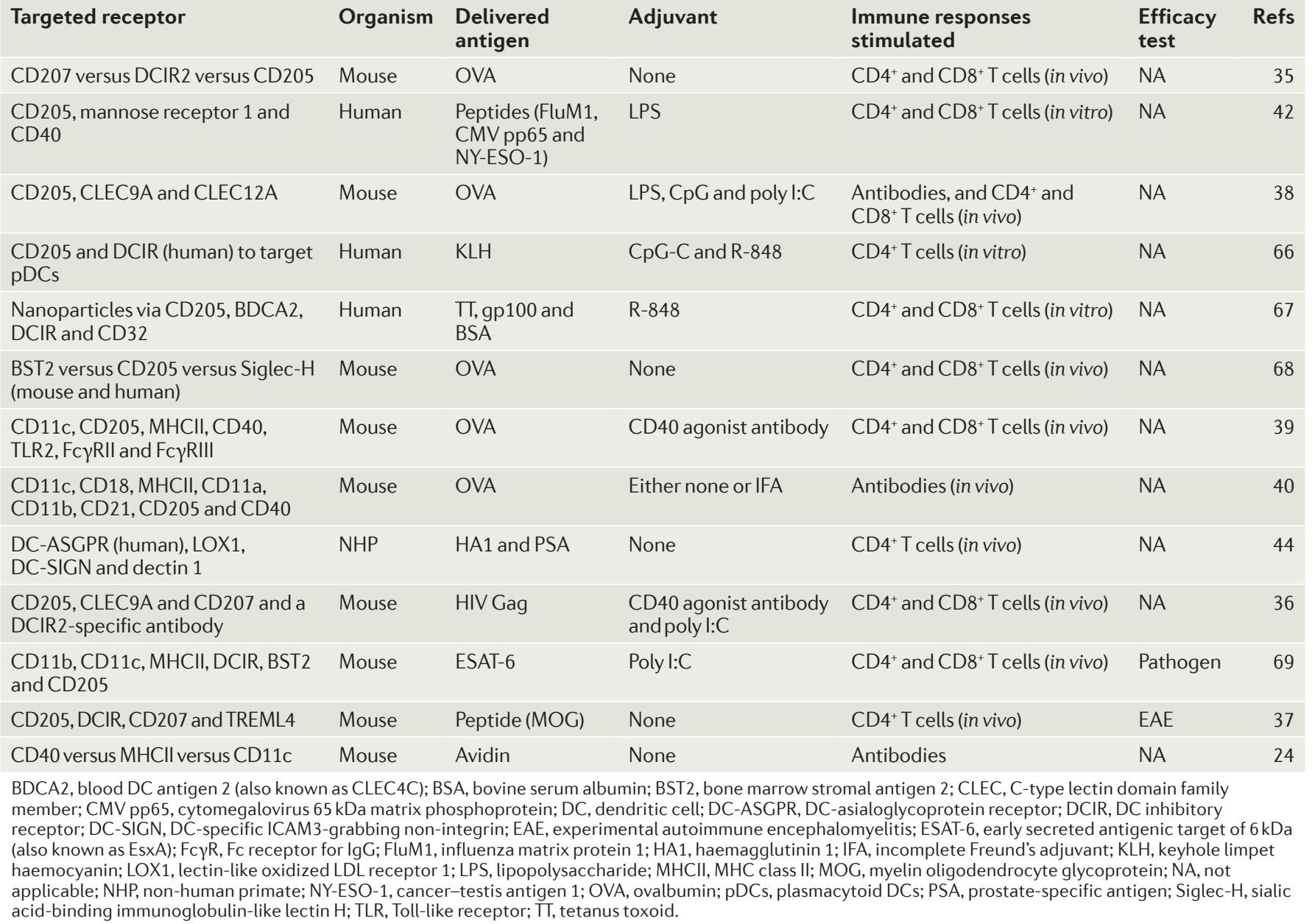

of an antibody response, it remains unclear how DC-targeted vaccines will be superior to current and more recently improved formulations of protein-based vaccines.

DC targeting to prime $T$ helper cell responses. As mentioned above, CD205 is the most widely studied DC target in mice, NHPs and humans. CD205 targeting (using HIV Gag with poly-ICLC) induced potent multifunctional $\mathrm{CD} 4^{+} \mathrm{T}$ cell cytokine responses in NHPs, but was not superior to an untargeted protein-based vaccine ${ }^{47}$. Although there are other receptors on several DC subsets that can induce potent $\mathrm{T}_{\mathrm{H}} 1$ cell responses (TABLE 3) (also see Supplementary information S1 (table)), there is little data beyond mouse studies that show any advantage over well-formulated protein-based vaccines.

DC targeting to induce CTL responses. The greatest challenge with non-live vaccine approaches is to induce potent, durable and protective CTL immunity. Even if DC-targeted vaccines do not turn out to be superior in terms of eliciting antibody and $\mathrm{CD} 4^{+} \mathrm{T}$ cell responses, CTL induction may still be the major advantage of this approach. Although the cell biological mechanisms of antigen cross-presentation (which is required for such a vaccine) are conserved between mice and humans, this function is largely restricted to the $\mathrm{CD} 8 \mathrm{a}^{+} \mathrm{DC}$ subset in mice, whereas it seems to be a general property of many human DC subsets ${ }^{43,48}$. However, data confirming that multiple human DC subsets can efficiently cross-present antigen in vivo are still lacking. In addition, recent single-cell RNA sequencing analysis suggests that multiple functional states (rather than pre-programmed functions) define specific DC subsets ${ }^{49}$, and these functional states may largely depend on the tissue microenvironment. Consistent with this, transcriptome-based network analysis of human macrophage activation showed that a simplistic dichotomous view (for example, M1 versus M2 macrophages) is insufficient to describe the multiple activation states of these cells $s^{50}$. As targeting strategies are usually aimed at surface receptors - the expression of which is only loosely associated with DC subsets and may change during activation we suggest that vaccines should be targeted to receptors that possess a functional role in a desired immune response. Such roles include stimulating favourable signalling pathways within the $\mathrm{DC}^{51}$, or introducing antigen into the correct intracellular compartment for antigen loading onto MHC class I or class II molecules ${ }^{30,42,52}$, as has been demonstrated for the mannose receptor 1, CLEC9A and CD40 (REFS 42,52,53).

At present, there is little evidence that DC-targeted or non-DC-targeted proteinbased vaccines will induce comparable or better CTL responses than those achieved by viral vaccines in humans. Nevertheless, the potential of this approach may lie in a combined heterologous prime-boost regimen, in which a low level CTL response induced by a non-live vaccine is strongly boosted by a viral vector ${ }^{47}$. In addition, heterologous prime-boost approaches with different viral vaccines induce potent CTL immunity 


\author{
Glossary \\ Adjuvants \\ Agents that are mixed with an antigen to increase the \\ immune response to that antigen following immunization. \\ Conjugate vaccines \\ Vaccines that consist of an antigen and an adjuvant that \\ are physically linked to allow for synchronous delivery of \\ both components. \\ Cross-presentation \\ $\mathrm{MHC}$ class I antigen presentation of antigens that are not \\ synthesized in the cytosol of a cell.

\section{C-type lectins} \\ Receptors that bind carbohydrates in a calcium-dependent \\ manner. They can be classified on the basis of their \\ signalling properties, which also influence the cellular \\ routing of the internalized receptor and subsequent antigen \\ presentation of the bound cargo.

\section{Fc $\gamma$ receptors} \\ ( $F(\gamma$ Rs). Receptors that specifically bind to the \\ crystallizable, non-antigen-binding part of IgG
}

that could be further boosted by targeted or non-targeted protein-based vaccines as they circumvent neutralizing anti-vector immunity. Moreover, for an immunotherapy or vaccine to be effective against cancer, additional treatments to limit ongoing immunosuppressive mechanisms within the patient may be necessary. Recent clinical studies have shown promising results using monoclonal antibodies to block the $\mathrm{T}$ cell inhibitory molecules cytotoxic T lymphocyte antigen 4 (CTLA4), and programmed cell death protein 1 (PD1) and its ligand PDL1 in vivo, thereby preventing negative regulation of the T cells ${ }^{54-56}$. Thus, combination therapies to increase CTL activation, prevent tumour-induced immunosuppression and modulate the tumour vasculature to enhance CTL infiltration ${ }^{57}$ may be crucial for successful cancer immunotherapy. Exactly how these antibodies need to be combined with DC-targeting approaches needs to be addressed in the future ${ }^{58}$.

\section{Conclusions}

In the past decade, $>100$ preclinical studies have analysed DC-targeting approaches that induce $\mathrm{T}$ cell and antibody responses, and most of these have been conducted in vivo in mice or in vitro using human cells (TABLES 2,3) (also see Supplementary information S1 (table)). Mouse studies have been, and will remain, crucial for gaining mechanistic insights into $\mathrm{T}$ cell priming - owing to the genetic manipulation and imaging techniques that are available - and they are therefore an important first step in vaccine development. antibodies. Binding via $\mathrm{F} c \gamma \mathrm{Rs}$ typically leads to internalization of the receptor and activation of the cell, thus enhancing phagocytosis and pathogen elimination.

Integrins

Transmembrane receptors that mediate attachments between cells or between cells and their surroundings for example. the extracellular matrix or blood vessels.

Pattern recognition receptor

(PRR). A protein that is expressed by innate immune cells that detects molecules associated with microbial pathogens or cellular stress.

\section{Toll-like receptor}

(TLR). An evolutionarily conserved pattern recognition receptor. These molecules are located intracellularly and also at the cell surface of macrophages, dendritic cells, $\mathrm{B}$ cells and intestinal epithelial cells. Their natural ligands are conserved molecular patterns - known as pathogenassociated molecular patterns - which are found in bacteria, viruses and fungi.

The crucial question at present is whether targeting specific DC subsets is more efficient than using well-formulated protein-based or particle-based vaccines with potent TLR adjuvants. Future studies need to compare these approaches using antigens from infectious pathogens or tumours, or combine them with viral vaccines to elucidate their potency. Such studies should use mouse models with more physiological antigens and then advance to NHPs for immunogenicity and ideally protection studies, as these provide greater predictive value before advancing to human studies.

In summary, based on the crucial need to improve $\mathrm{T}$ cell immunity, the elegant research on DC-targeting vaccines has provided sufficient mechanistic insights and encouraging functional evidence to vigorously move the field forward and determine whether DC targeting can fulfil its promise in humans to improve protection against infection and tumours.

Wolfgang Kastenmüller is at the Institute of Molecular Medicine, University of Bonn, 53105 Bonn, Germany.

Kathrin Kastenmüller is at the Institute of Experimental Immunology and the Department of General Practice and Family Medicine, University of Bonn, 53105 Bonn,

Germany.

Christian Kurts is at the Institute of Experimental Immunology, University of Bonn, 53105 Bonn,

Germany.

Robert A. Seder is at the Cellular Immunology Section, Vaccine Research Center, National Institute of Allergy and Infectious Diseases, Bethesda Maryland 20892-3005, USA.

Correspondence to R.A.S. and W.K. e-mails: rseder@mail.nih.gov; wkastenm@uni-bonn.de doi:10.1038/nri3727 Published online 5 September 2014
1. Plotkin, S. A. Vaccines: correlates of vaccine-induced immunity. Clin. Infect. Dis. 47, 401-409 (2008). 2. Tomaras, G. D. \& Haynes, B. F. Advancing toward HIV-1 vaccine efficacy through the intersections of immune correlates. Vaccines 2, 15-35 (2014).

3. de Souza, J. B. Protective immunity against malaria after vaccination. Parasite Immunol. 36, 131-139 (2014).

4. Andersen, P. \& Woodworth, J. S. Tuberculosis vaccines - rethinking the current paradigm. Trends Immunol. 35, 387-395 (2014).

5. Guermonprez, P., Valladeau, J., Zitvogel, L., Thery, C. $\Sigma$ Amigorena, S. Antigen presentation and T cell stimulation by dendritic cells. Annu. Rev. Immunol. 20, 621-667 (2002).

6. Schuler, G., Schuler-Thurner, B. \& Steinman, R. M The use of dendritic cells in cancer immunotherapy. Curr. Opin. Immunol. 15, 138-147 (2003).

7. Palucka, K. \& Banchereau, J. Cancer immunotherapy via dendritic cells. Nature Rev. Cancer 12, 265-277 (2012).

8. Palucka, A. K. et al. Dendritic cells loaded with killed allogeneic melanoma cells can induce objective clinical responses and MART-1 specific CD8 ${ }^{+}$T-cell immunity. J. Immunother. 29, 545-557 (2006).

9. Frankenberger, B. \& Schendel, D. J. Third generation dendritic cell vaccines for tumor immunotherapy. Eur. J. Cell Biol. 91, 53-58 (2012).

10. Wimmers, F., Schreibelt, G., Skold, A. E., Figdor, C. G. $\&$ De Vries, I. J. Paradigm shift in dendritic cell-based immunotherapy: from in vitro generated monocytederived DCs to naturally circulating DC subsets. Front. Immunol. 5, 165 (2014).

11. Blander, J. M. \& Medzhitov, R. Toll-dependent selection of microbial antigens for presentation by dendritic cells. Nature 440, 808-812 (2006).

12. Sporri, R. \& Reis e Sousa, C. Inflammatory mediators are insufficient for full dendritic cell activation and promote expansion of CD4+ $\mathrm{T}$ cell populations lacking helper function. Nature Immunol. 6, 163-170 (2005).

13. Wong, P. \& Pamer, E. G. CD8 T cell responses to infectious pathogens. Annu. Rev. Immunol. 21, 29-70 (2003).

14. Reed, S. G., Bertholet, S., Coler, R. N. \& Friede, M. New horizons in adjuvants for vaccine development. Trends Immunol. 30, 23-32 (2009).

15. Coffman, R. L., Sher, A. \& Seder, R. A. Vaccine adjuvants: putting innate immunity to work. Immunity 33, 492-503 (2010).

16. Cho, H. J. et al. Immunostimulatory DNA-based vaccines induce cytotoxic lymphocyte activity by a T-helper cell-independent mechanism. Nature Biotechnol. 18, 509-514 (2000)

17. Tighe, H. et al. Conjugation of protein to immunostimulatory DNA results in a rapid, long-lasting and potent induction of cell-mediated and humoral immunity. Eur. J. Immunol. 30, 1939-1947 (2000).

18. Tighe, H. et al. Conjugation of immunostimulatory DNA to the short ragweed allergen Amb a 1 enhances its immunogenicity and reduces its allergenicity. J. Allergy Clin. Immunol. 106, 124-134 (2000).

19. Wille-Reece, U., Wu, C.-Y., Flynn, B. J., Kedl, R. M. \& Seder, R. A. Immunization with HIV-1 Gag protein conjugated to a TLR7/8 agonist results in the generation of HIV-1 Gag-specific Th 1 and CD8 ${ }^{+} \mathrm{T}$ cell responses. J. Immunol. 174, 7676-7683 (2005)

20. Kastenmüller, K. et al. Protective T cell immunity in mice following protein-TLR7/8 agonist-conjugate immunization requires aggregation, type I IFN, and multiple DC subsets. J. Clin. Invest. 121, 1782-1796 (2011).

21. Wille-Reece, U. et al. HIV Gag protein conjugated to a Toll-like receptor $7 / 8$ agonist improves the magnitude and quality of Th1 and CD8 $8^{+} \mathrm{T}$ cell responses in nonhuman primates. Proc. Natl Acad. Sci. USA 102, 15190-15194 (2005)

22. Snider, D. P. \& Segal, D. M. Targeted antigen presentation using crosslinked antibody heteroaggregates. J. Immunol. 139, 1609-1616 (1987).

23. Carayanniotis, G. $\&$ Barber, B. H. Adjuvant-free IgC responses induced with antigen coupled to antibodies against class II MHC. Nature 327, 59-61 (1987).

24. Frleta, D., Demian, D. \& Wade, W. F. Class II-targeted antigen is superior to CD40-targeted antigen at stimulating humoral responses in vivo. Int. Immunopharmacol. 1, 265-275 (2001).

25. Tewari, K. et al. Poly(l:C) is an effective adjuvant for antibody and multi-functional CD4 ${ }^{+} \mathrm{T}$ cell responses to Plasmodium falciparum circumsporozoite protein (CSP) and $\alpha$ DEC-CSP in non human primates. Vaccine 28, 7256-7266 (2010). 
26. Bachem, A. et al. Superior antigen cross-presentation and XCR 1 expression define human $C D 11 \mathrm{C}^{+} \mathrm{CD} 141^{+}$ cells as homologues of mouse CD8 ${ }^{+}$dendritic cells. J. Exp. Med. 207, 1273-1281 (2010).

27. Crozat, K. et al. The XC chemokine receptor 1 is a conserved selective marker of mammalian cells homologous to mouse $\mathrm{CD} 8 \alpha^{+}$dendritic cells. J. Exp. Med. 207, 1283-1292 (2010).

28. Jongbloed, S. L. et al. Human CD $141^{+}\left(\right.$BDCA-3) ${ }^{+}$ dendritic cells (DCs) represent a unique myeloid DC subset that cross-presents necrotic cell antigens. J. Exp. Med. 207, 1247-1260 (2010).

29. Poulin, L. F. et al. Characterization of human DNGR-1 $\mathrm{BDCA3}$ + leukocytes as putative equivalents of mouse CD8 $\alpha^{+}$dendritic cells. J. Exp. Med. 207, 1261-1271 (2010).

30. Sancho, D. et al. Tumor therapy in mice via antigen targeting to a novel, DC-restricted C-type lectin. J. Clin. Invest. 118, 2098-2110 (2008)

31. Joffre, O. P., Sancho, D., Zelenay, S., Keller, A. M. \& Reis e Sousa, C. Efficient and versatile manipulation of the peripheral CD4 ${ }^{+}$T-cell compartment by antigen targeting to DNGR-1/CLEC9A. Eur. J. Immunol. 40 1255-1265 (2010).

32. Mascarell, L. et al. Delivery of the HIV-1 Tat protein to dendritic cells by the CyaA vector induces specific Th 1 responses and high affinity neutralizing antibodies in non human primates. Vaccine 24, 3490-3499 (2006).

33. Mascarell, L., Fayolle, C., Bauche, C., Ladant, D. \& Leclerc, $C$. Induction of neutralizing antibodies and Th1-polarized and CD4-independent CD8 ${ }^{+}$T-cell responses following delivery of human immunodeficiency virus type 1 Tat protein by recombinant adenylate cyclase of Bordetella pertussis. J. Virol. 79, 9872-9884 (2005)

34. Steinman, R. M. \& Banchereau, J. Taking dendritic cells into medicine. Nature 449, 419-426 (2007).

35. Idoyaga, J. et al. Cutting edge: langerin/CD207 receptor on dendritic cells mediates efficient antigen presentation on $\mathrm{MHCI}$ and II products in vivo. J. Immunol. 180, 3647-3650 (2008).

36. Idoyaga, J. et al. Comparable Thelper 1 (Th1) and CD8 T-cell immunity by targeting HIV gag p 24 to CD8 dendritic cells within antibodies to Langerin DEC205, and Clec9A. Proc. Natl Acad. Sci. USA 108, 2384-2389 (2011).

37. Idoyaga, J. et al. Specialized role of migratory dendritic cells in peripheral tolerance induction. J. Clin. Invest. 123, 844-854 (2013).

38. Lahoud, M. H. et al. Targeting antigen to mouse dendritic cells via Clec9A induces potent CD4 T cell responses biased toward a follicular helper phenotype. J. Immunol. 187, 842-850 (2011).

39. Castro, F. V. V. et al. CD 11 c provides an effective immunotarget for the generation of both CD4 and CD8 T cell responses. Eur. J. Immunol. 38, 2263-2273 (2008).

40. White, A. L. et al. Ligation of CD11c during vaccination promotes germinal centre induction and robust humoral responses without adjuvant. Immunology 131, 141-151 (2010).

41. Van Montfoort, N. et al. Circulating specific antibodies enhance systemic cross-priming by delivery of complexed antigen to dendritic cells in vivo. Eur. J. Immunol. 42, 598-606 (2012).

42. Chatterjee, B. et al. Internalization and endosomal degradation of receptor-bound antigens regulate the efficiency of cross presentation by human dendritic cells. Blood 120, 2011-2020 (2012).

43. Cohn, L. et al. Antigen delivery to early endosomes eliminates the superiority of human blood BDCA3 dendritic cells at cross presentation. J. Exp. Med. 210, 1049-1063 (2013).

44. Li, D. et al. Targeting self- and foreign antigens to dendritic cells via DC-ASGPR generates IL-10-producing suppressive CD4 ${ }^{+} \mathrm{T}$ cells J. Exp. Med. 209, 109-121 (2012).

45. Valladeau, J. et al. Immature human dendritic cells express asialoglycoprotein receptor isoforms for efficient receptor-mediated endocytosis. J. Immunol. 167, 5767-5774 (2001)

46. Kreutz, M., Tacken, P. J. \& Figdor, C. G. Targeting dendritic cells-why bother? Blood 121, 2836-2844 (2013).

47. Flynn, B. J. et al. Immunization with HIV Gag targeted to dendritic cells followed by recombinant New York vaccinia virus induces robust T-cell immunity in nonhuman primates. Proc. Natl Acad. Sci. USA 108, 7131-7136 (2011).
48. Segura, E. \& Amigorena, S. Cross-presentation by human dendritic cell subsets. Immunol. Lett. 158, 73-78 (2013)

49. Jaitin, D. A. et al. Massively parallel single-cell RNA-seq for marker-free decomposition of tissues into cell types. Science 343, 776-779 (2014).

50. Xue, J. et al. Transcriptome-based network analysis reveals a spectrum model of human macrophage activation. Immunity 40, 274-288 (2014)

51. Gross, O. et al. Card9 controls a non-TLR signalling pathway for innate anti-fungal immunity. Nature 442, 651-656 (2006)

52. Burgdorf, S., Kautz, A., Bohnert, V., Knolle, P. A. \& Kurts, C. Distinct pathways of antigen uptake and intracellular routing in CD4 and CD8 T cell activation. Science 316, 612-616 (2007).

53. Sancho, D. et al. Identification of a dendritic cell receptor that couples sensing of necrosis to immunity. Nature 458, 899-903 (2009).

54. Brahmer, J. R. et al. Safety and activity of anti-PD-L1 antibody in patients with advanced cancer. N. Engl. J. Med. 366, 2455-2465 (2012).

55. Topalian, S. L. et al. Safety, activity, and immune correlates of anti-PD-1 antibody in cancer. N. Engl. J. Med. 366, 2443-2454 (2012).

56. Honda, T. et al. Tuning of antigen sensitivity by T cell receptor-dependent negative feedback controls $\mathrm{T}$ cell effector function in inflamed tissues. Immunity $\mathbf{4 0}$ 235-247 (2014).

57. Hamzah, J et al. Vascular normalization in Rgs5-deficient tumours promotes immune destruction. Nature 453, 410-414 (2008)

58. Pardoll, D. M. The blockade of immune checkpoints in cancer immunotherapy. Nature Rev. Cancer 12. 252-264 (2012)

59. Heit, A. et al. Cutting edge: Toll-like receptor 9 expression is not required for $\mathrm{CpG}$ DNA-aided cross-presentation of DNA-conjugated antigens but essential for cross-priming of CD8 T cells. J. Immunol. 170, 2802-2805 (2003).

60. Horner, A. A. et al. Immunostimulatory DNA-based vaccines elicit multifaceted immune responses against HIV at systemic and mucosal sites. J. Immunol. 167, 1584-1591 (2001)

61. Heit, A. et al. Protective CD8 T cell immunity triggered by $\mathrm{CpG}$-protein conjugates competes with the efficacy of live vaccines. J. Immunol. 174 4373-4380 (2005)
62. Heit, A. et al. Circumvention of regulatory $\mathrm{CD} 4^{+} \mathrm{T}$ cell activity during cross-priming strongly enhances $T$ cellmediated immunity. Eur. J. Immunol. 38, 1585-1597 (2008).

63. Huleatt, J. W. et al. Vaccination with recombinant fusion proteins incorporating Toll-like receptor ligands induces rapid cellular and humoral immunity. Vaccine 25, 763-775 (2007).

64. Wang, B. et al. A Toll-like receptor-2-directed fusion protein vaccine against tuberculosis. Clin. Vaccine Immunol. 14, 902-906 (2007).

65. Jackson, D. C. et al. A totally synthetic vaccine of generic structure that targets Toll-like receptor 2 on dendritic cells and promotes antibody or cytotoxic T cell responses. Proc. Natl Acad. Sci. USA 101, 15440-15445 (2004).

66. Tel, J. et al. DEC-205 mediates antigen uptake and presentation by both resting and activated human plasmacytoid dendritic cells. Eur. J. Immunol. 41 1014-1023 (2011)

67. Tel, J. et al. Targeting uptake receptors on human plasmacytoid dendritic cells triggers antigen cross-presentation and robust type I IFN secretion. J. Immunol. 191, 5005-5012 (2013).

68. Moffat, J. M. et al. Targeting antigen to bone marrow stromal cell-2 expressed by conventional and plasmacytoid dendritic cells elicits efficient antigen presentation. Eur. J. Immunol. 43, 595-605 (2013)

69. Dong, H. et al. Induction of protective immunity against Mycobacterium tuberculosis by delivery of ESX antigens into airway dendritic cells. Mucosal Immunol. 6, 522-534 (2013).

\section{Acknowledgements}

W.K. and C.K. are members of the Deutsche Forschungsgemeinschaft Excellence Cluster ImmunoSensation in Bonn Germany, and are supported by grant SFB704. W.K. is supported by the NRW-Rückkehrerprogramm of the German state of Northrhine-Westfalia. The authors thank G. Gasteiger, $\mathrm{N}$. Garbi and their laboratory members for critically reading this manuscript.

Competing interests statement

The authors declare no competing interests.

SUPPLEMENTARY INFORMATION

See online article: $\underline{\mathrm{S}}$ (table)

ALL LINKS ARE ACTIVE IN THE ONLINE PDF 